\title{
The Development of Cataract Following Trabeculectomy
}

\author{
MICHAEL P. CLARKE, STEPHEN A. VERNON, JAMES H. SHELDRICK
}

Nottingham

\begin{abstract}
Summary
Objective-To determine the risk of cataract development following trabeculectomy.

Design-A prospective follow-up study of patients seen pre-operatively and at one, three, six and 12 months following surgery and subsequently at yearly intervals. Setting-Ophthalmology Department of Nottingham University Hospital.

Patients-Fifty-seven patients with primary open angle glaucoma, including pseudoexfoliation, undergoing trabeculectomy.

Main outcome measures-Increases in lens opacity measured by the Lens Opacity Meter 701, the Keeler Projectoscope and clinical grading.

Results-Of 35 patients seen at six months post-operatively, none had lost two Snellen lines of visual acuity due to lens opacity, although three patients showed evidence of increasing lens opacity on all three parameters.

Conclusion-There is no evidence of visually significant lens opacity occurring at six months post-operative follow-up.
\end{abstract}

There is an increasing trend among British ophthalmologists to opt for early surgical treatment of primary open angle glaucoma. Smith, ${ }^{1}$ in a long term study of medical versus surgical treatment for glaucoma found drainage surgery to be superior to medical therapy in lowering intra-ocular pressure, but a higher incidence of cataract in the surgically treated group. Trabeculectomy, popularised by the late John Cairns, ${ }^{2}$ has been shown to be a much safer procedure than its predecessors, and the recent report by Jay et $a l^{3}$ has confirmed the clinical impression of many surgeons that early surgery, i.e. surgery performed when the diagnosis is confirmed, rather than when medical treatment has been shown to be inadequate to prevent disease progression, has a beneficial effect on outcome.
If this approach is to be adopted, it is important that operative complications are minimal. There is a fear that trabeculectomy hastens the development of cataract, particularly in the elderly.

The incidence of cataract formation following glaucoma surgery is estimated at between four and $38 \%$ on the basis of retrospective studies. ${ }^{4-8}$ The causes of cataract development after trabeculectomy are not known. Subsequent cataract surgery may compromise filtration. It is desirable that an accurate figure for this complication is available to surgeons which takes into account the presence of preexisting lens opacity. This information would be useful when combined cataract and filtration surgery is being considered.

We are undertaking a prospective study to try and further define the incidence of cataract

From: Academic Unit of Ophthalmology, Queen's Medical Centre, Nottingham.

Correspondence to Mr Clarke, Department of Ophthalmology, Hospital for Sick Children, 555, University Ave., Toronto, Ontario, Canada M5G 1 X8. 
following trabeculectomy and the risk factors involved.

\section{Patients}

Fifty-seven patients about to undergo trabeculectomy for chronic open angle glaucoma have been recruited to date. Approval for the study was obtained from the local ethical committee and informed consent was obtained from all participants, three have defaulted. Of the remaining 54, 38 are male and the age range is from 31 to 89 years (mean 66 years). Seven have pseudoexfoliation and one has pigmentary glaucoma. Twelve patients have had bilateralsurgery. Fifty patients require no additional treatment to control intraocular pressure following surgery. No patient has required any further surgical procedure following trabeculectomy. The results from the 35 patients for whom six month follow-up is available are reported.

\section{Methods}

\section{Measurement of Lens Opacity}

Lens opacity may be quantified using a number of techniques including clinical grading, ${ }^{9}$ target projection ophthalmoscopy, ${ }^{10}$ retroillumination photography and densitometry, measurement of light back scattered from defined regions of the lens, microdensitometry of Scheimpflug photographs ${ }^{11}$ and commerically available lensometers. ${ }^{12}$

We have chosen to use clinical grading, target projection ophthalmoscopy using the Keeler Projectoscope, and a commerically available lensometer, the Lens Opacity Meter 701.

The Lens Opacity Meter 701 (Interzeag, Switzerland) assesses lens density by measuring the scattering of a $1.5 \mathrm{~mm}$ beam of $700 \mathrm{~nm}$ wavelength directed along the visual axis. ${ }^{12,13}$ The scattered light is detected by a photocell $27^{\circ}$ below the incident beam. The instrument provides a quick, reproducible, numerical measure of the degree of lens opacity which correlates well with slit lamp grading. Although it is possible to make accurate measurements through an undilated pupil, the manufacturers recommend that the pupil diameter should be at least $4 \mathrm{~mm}$.

The Keeler Projectoscope ${ }^{10}$ projects 11, three bar RAF targets of increasing size onto the fundus. The smallest grating which could be resolved was recorded.

Clinical grading included assessment of the degree of cortical vacuoles, waterclefts and spokes, nucleus colour and density and subcapsular opacity.

For each patient, the following items of information were recorded: The presence of any systemic disease, systemic and ocular drug therapy, refraction and best corrected visual acuity, previous uveitis, and the presence of iris atrophy, pseudoexfoliation or posterior synechiae. Patients with diabetes, evidence of previous episodes of uveitis and those taking systemic steroids were excluded.

The pupils were dilated with tropicamide $1 \%$ and lens opacity was graded clinically, and measured with the Keeler Projectoscope and the Lens Opacity Meter.

Five measurements of lens density were made with the lens opacity meter, and a mean and standard deviation were calculated. The pupil size was recorded. Intra-operative complications and the use of any intraocular injection were recorded.

Post-operatively, the anterior chamber depth was measured with a Haag Streit pachymeter, and the presence of hyphaema or air in the anterior chamber recorded.

The patients were seen for review at one, three, six and 12 months post-operatively, when the refraction, best corrected acuity, intra-ocular pressure, anterior chamber depth, and bleb profile were recorded. The lens density was assessed clinically and with the Projectoscope and Lens Opacity Meter on each occasion after pupillary dilation. Postoperative readings were made without prior knowledge of previous measurements.

\section{Results}

The results from the 35 patients for whom six months follow-up is available are reported. Where bilateral surgery had been performed, the results are taken from the eye with the longer follow-up.

There were two intra-operative complications; one patient had a partial avulsion of the scleral trapdoor which was resewn, and one patient had a broad iridectomy performed instead of a peripheral iridectomy because of a dilated pupil at the time of surgery. 
At examination between 24 and 48 hours post-operatively, no visible lens injury was apparent in any patient. Twenty of the 35 had a macroscopic hyphaema at this stage. The anterior depth measurements recorded at this time are shown in Figure 1. No patient had a flat anterior chamber, although three had central anterior chamber depth of less than $1 \mathrm{~mm}$. All of these patients showed post-operative increases in lens opacity.

At the six month follow-up examination, none of the patients was taking topical or systemic medication to control the intraocular pressure, although three patients with non functional drainage blebs had pressures greater than $20 \mathrm{mmHg}$ (Figure 2). No patient had undergone any further surgical procedure to control the intra-ocular pressure.

\section{Lens Opacity Changes}

\section{Lens opacity meter}

Five readings were taken with the LOM preand post-operatively, and a mean and standard deviation calculated.

For an individual patient an increase of two standard deviations from the pre-operative reading was taken to signify an increased lens opacity post-operatively. On this basis, 18 of

Anterior Chamber Depth ( $\mathrm{mm}$.)

24-48 hours Postoperatively

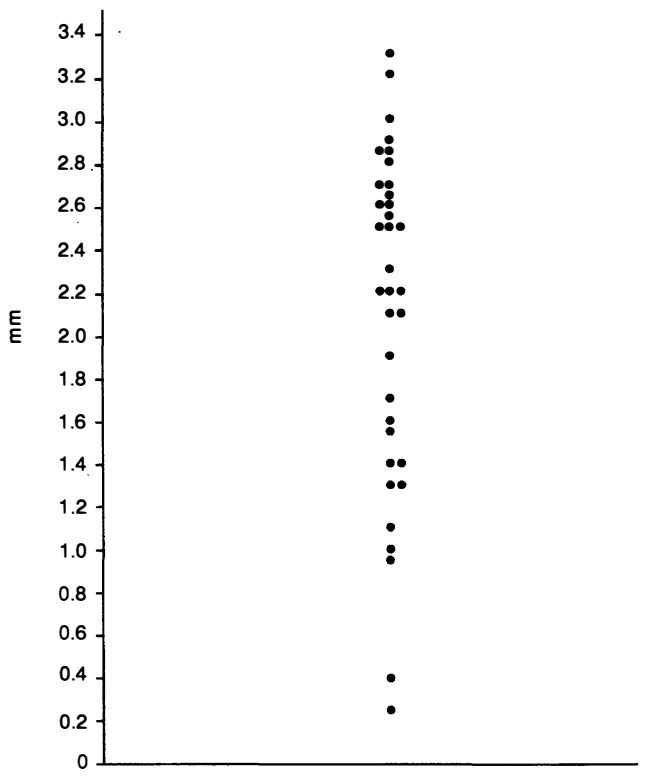

Fig. 1. the $35(51 \%)$ patients showed an increased lens opacity at six months follow-up. Four showed a significant decrease in lens opacity (Figure 3). The patient with the highest meter reading showed the most marked increase in lens opacity. Of 15 unoperated fellow eyes, six $(40 \%)$ showed significant increases in LOM readings, with none improving. This difference is not statistically significant. Taking the whole data set, there was also no significant difference between pre- and six month postoperative meter readings (Fisher Exact test). Using the data contained in the article by De Natale ${ }^{13}$ it is possible to calculate an age adjusted normal range for Lens Opacity Meter reading. The figure shows the $95 \%$ confidence limits calculated from readings on 266 normal subjects. It can be seen that five patients in our study had lens opacities above the normal range pre-operatively (Figure 4). At six months post-operatively, five remained above the normal range (Figure 5). No further patient had fallen outside the normal range, despite 18 of the 35 showing a more than two standard deviation increase from the mean of five pre-operative LOM readings six months post-operatively.

Intraocular Pressure In Operated Eyes (mmHg) at 6 months Post Operative

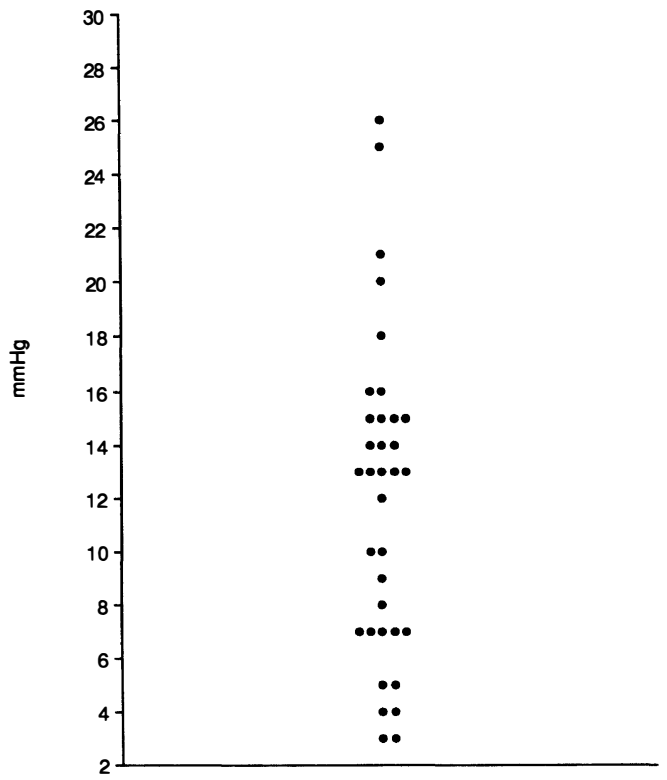

Fig. 2. 


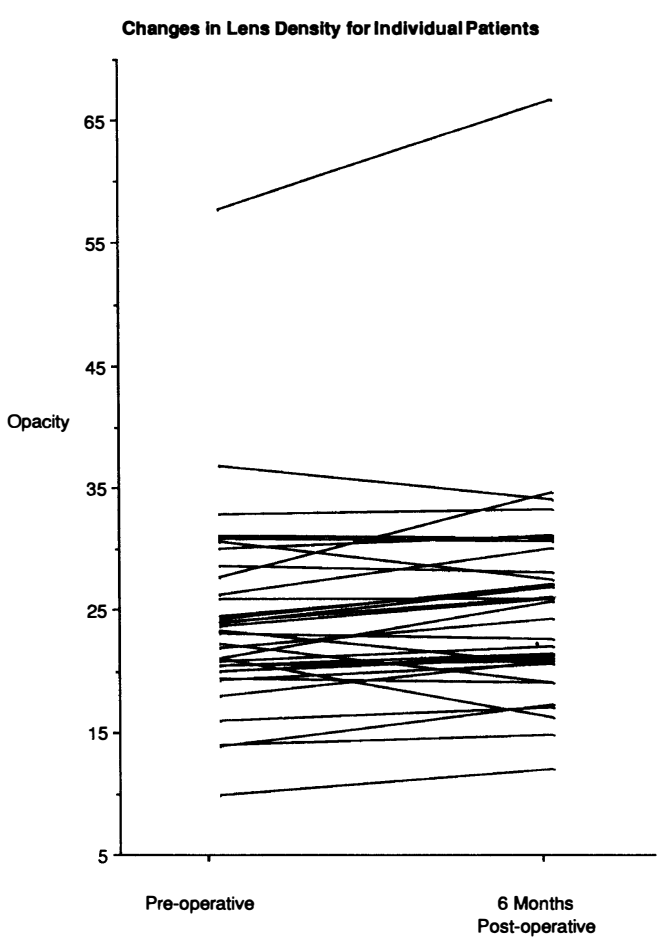

Fig. 3.

\section{Projectoscope Measurements}

Nine $(26 \%)$ patients showed evidence of increased lens opacity at six months, while four $(11 \%)$ showed a reduction in lens opacity as measured by any change in the smallest visible target. Three of the $15(20 \%)$ fellow unoperated eyes showed increased projectoscope measurements.

\section{Clinical Grading}

On the basis of any alteration in the grading scores, $15(43 \%)$ patients showed an increase in lens opacity clinically, with seven improving. Seven fellow eyes $(47 \%)$ deteriorated clinically.

\section{Agreement Between Different Methods of} Assessing Lens Opacity

Three patients $(8.6 \%)$ showed a worsening of lens opacity by all three methods. Ten patients $(29 \%)$ showed increases in both Lens Opacity Meter reading and grading scores, and four $(11 \%)$ showed increased LOM and projectoscope readings. Seven patients $(20 \%)$ showed increased grading scores and Projectoscope readings without significant increases in LOM readings.

\section{Pre Operative Lens Density vs. Age}

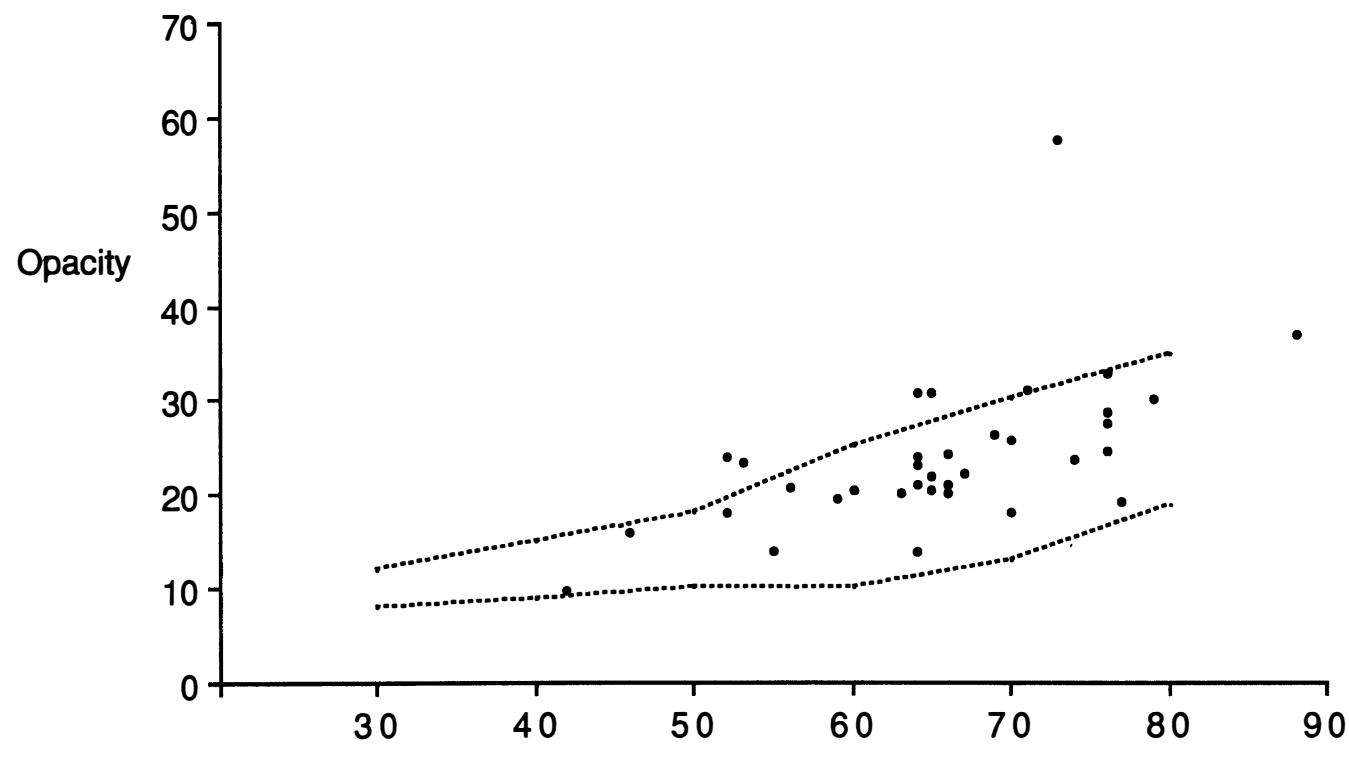

Fig. 4. 


\section{Lens Density vs. Age 6 months Post Surgery}

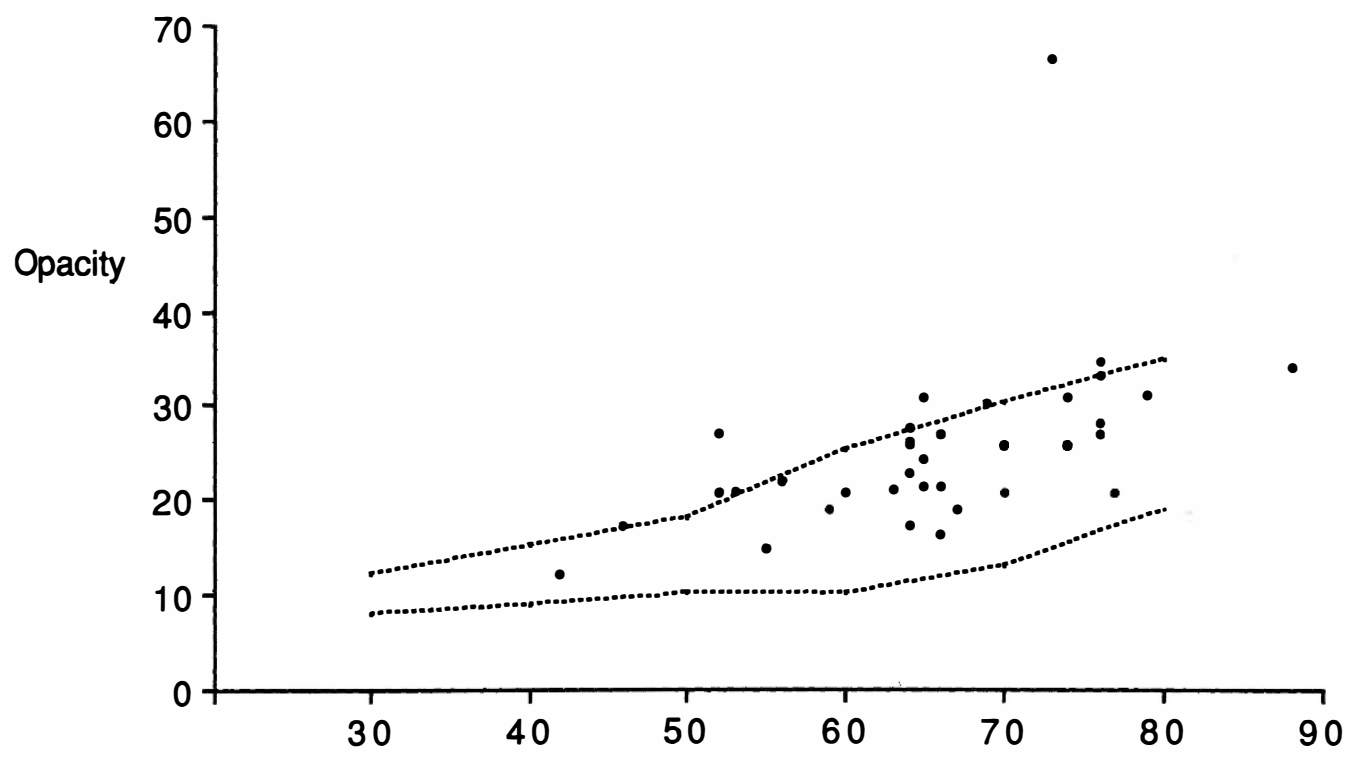

Age

Fig. 5.

Changes in Visual Acuity

Five patients $(14 \%)$ showed a drop in best corrected visual acuity of two Snellen lines or more post-operatively. All of these patients had preserved central field. Only one of these showed an increase in lens opacity as measured by all three parameters, and even this patient still retained an LOM reading within the normal range. In this patient, and in three of the other four, the intra-ocular pressure was $7 \mathrm{mmHg}$ or less post-operatively. All four patients showed clinical evidence of hypotony, and fluorescein angiograms performed in two patients showed evidence of choroidal folds and cystoid macular oedema (Figure 6) which may have made a significant contribution to the reduction in acuity.

No patient showed a two Snellen line or more improvement in best corrected visual acuity following surgery.

\section{Discussion}

A reasonable estimate of the prevalence of cataract after trabeculectomy, based on the published literature, is between ten and $20 \%$ depending on the definition of cataract used. This figure increases with increasing length of follow-up, but is less than the $40 \%$ found by Greve in a ten year prospective follow-up of the Double Flap Scheie operation. ${ }^{14}$

Our study indicates lens changes at six months in between eight and $29 \%$ of eyes at

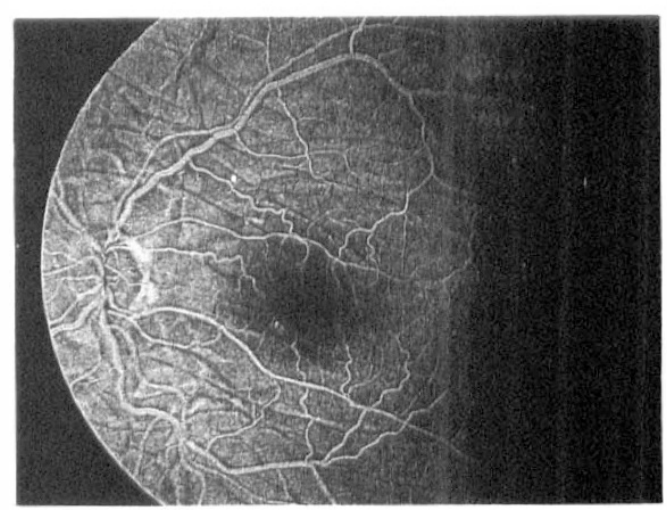

Fig. 6. Fluorescein angiogram of a patient in whom best corrected vision deteriorated from $6 / 9$ preoperatively to $6 / 18$ six months post-surgery showing choroidal folds. 
six months depending on the methods of assessment used.

The causes of cataract after trabeculectomy are unknown. It does not seem that patients with glaucoma are more likely to develop cataract than normal controls. ${ }^{15}$ Medical treatment of glaucoma may be cataractogenic, as was shown to be the case with phospholine iodide in the 1960s. ${ }^{16}$

Priestley Smith in the last century noted acute cataract formation as a complication of glaucoma surgery and attributed this to direct trauma to the lens. ${ }^{17}$ A number of such cases have been reviewed histologically ${ }^{18}$ and in a more recent paper, ${ }^{4}$ two patients developed cataract immediately following surgery.

Direct surgical trauma apart, what is it that predisposes an eye to develop cataract following a trabeculectomy? Retrospective studies have identified two factors; a flat anterior chamber with lens-corneal touch, and hypotony, ${ }^{4,5}$ although the mechanisms are obscure. The method of trabeculectomy may also be relevant, a higher incidence of lens opacity progression being found when the meshwork is excised from anterior to posterior. ${ }^{19}$ Whether eyes with pre-existing lens opacity are more or less likely to show progression lens opacity is disputed. ${ }^{3,20}$ Our results indicate those with preexisting lens opacity to be more at risk, although more follow-up is required to demonstrate this statistically. It may be that alterations in aqueous humour dynamics following trabeculectomy have an adverse effect on lens metabolism.

The patients in this study are necessarily preselected, as some surgeons in this unit routinely perform combined surgery on patients with significant lens opacity. We have made an attempt to quantify lens opacity prior to surgery. This is important because reduction of visual acuity alone is not an adequate measure of lens opacity. Marked increases in nucleus density can occur with preservation of corrected acuity, and conversely we have seen marked reduction of visual acuity in a few of our patients who show no evidence of progression of lens opacity.

The methods we have chosen to assess lens opacity have some drawbacks. One of the major advantages of the Lens Opacity Meter is that it gives a reproducible, objective, numerical result which allows instant comparison between measurements taken at different times. The results are, however expressed in machine units, and while our investigations into this point are not yet complete, it appears that the readings mainly reflect nucleus density, and may be within normal limits in patients with a clear nucleus but significant posterior subcapsular opacity.

The Projectoscope is prone to overestimate lens opacity in patients with refractive errors, particularly astigmatism. In addition, in patients with inhomogeneous lens opacity, it is often possible to look through gaps and obtain a normal measurement.

We have seen three patients in our series with hypotonic eyes who have lost 2 Snellen lines of acuity and in whom there is no evidence of cataract progression. This is not a widely reported finding, although Watson ${ }^{19}$ mentions one patient with a reduction in pressure from $54 \mathrm{mmHg}$ pre-operatively to $3 \mathrm{mmHg}$ post-operatively in whom poor vision post-operatively was attributed to low pressure.

We have been able to demonstrate subtle changes in lens opacity by three independent methods in $8.6 \%$ of our patients $(95 \%$ confidence limits $0-17.9 \%$ ) at six months following trabeculectomy. It is, however, important to emphasise that in no case have these changes caused a reduction in visual acuity and it is possible that many will prove to be non-progressive as the study continues. We plan to extend the study to include a control group of medically treated patients to determine more fully the risks of cataract formation in patients offered trabeculectomy.

The authors would like to thank the consultant staff at Nottingham University Hospital for permission to study patients under their care.

\section{References}

${ }^{1}$ Smith RJH: The Enigma of Primary Open Angle Glaucoma. Trans. Ophthalmol Soc UK 1986, 105: 618-33.

${ }^{2}$ Cairns JE: Trabeculectomy: Preliminary report of a new method. Am J Ophthalmol 1968, 66: 673-9.

${ }^{3}$ Jay JL and Murray SB: Early trabeculectomy versus conventional management in primary open angle glaucoma. Br J Ophthalmol 1988, 72: 881-89.

${ }^{4}$ D'Ermo F, Bonomi L, Doro D: Critical analysis of the long term results of trabeculectomy. Am J Ophthalmol 1979, 88: 829-35.

${ }^{5}$ Mills KB: Trabeculectomy: a retrospective long 
term follow-up of 444 cases. $\mathrm{Br} J$ Ophthalmol 1981, 65: 790-5.

${ }^{6}$ Sugar HS: Post-operative cataract in successfully filtering glaucomatous eyes. Am J Ophthalmol 1970, 69: 740-6.

${ }^{7}$ Ridgway AEA: Trabeculectomy-a follow-up study. Br J Ophthalmol 1974, 58: 680-6.

${ }^{8}$ Jerndal T and Lundstrom M: 300 trabeculectomies followed up for three years. Acta Ophthalmol 1977, 55: 52-62.

${ }^{9}$ Sparrow JM, Bron AI, Brown NAP, Ayliffe W, Hill AR: The Oxford clinical cataract classification and grading system. Int Ophthalmol 1986, 9: 207-25.

${ }^{10}$ Cutlier E, Fagadu W, Cicchetti DV: Methods of Evaluation of Medical Therapy of Senile and Diabetic Cataracts. Trans Ophthalmol Soc UK 1982, 102: 416-22.

${ }^{11}$ Hockwin O, Dragomirescu V, Laser H: Age related changes in normal and cataractous human lens density obtained by microdensitometric image analysis of Scheimpflug photographs. Lens Res 1983, 1: 207-20.

${ }^{12}$ Flammer $\mathbf{J}$ and Bebie H: Lens Opacity Meter: A New Instrument to Quantify Lens Opacity. Ophthalmologica 1987, 195: 69-72.
${ }^{13}$ De Natale R, Flammer J, Zulauf M, Bebie T: Influence of age on the transparency of the lens in normals: A population study with help of the Lens Opacity Meter 701. Ophthalmologica 1988, 196: 14-18.

${ }^{14}$ Greve EL, Dake CL, Klaver JHJ, Mutsaerts EMG: Ten year prospective follow-up of a glaucoma operation. The double flap Scheie in primary open angle glaucoma. Int Ophthalmol 1985, 8: 139-46.

${ }^{15}$ Schaffer RN and Rosenthal G: Comparison of cataract incidence in normal and glaucomatous population. Am J Ophthalmol 1970, 69: 368-70.

${ }^{16}$ Axelsson $\mathrm{V}$ and Holmberg A: The Frequency of Cataract after miotic therapy. Acta Ophthalmol 1966, 44: 421-9.

${ }^{17}$ Smith P: Pathology and treatment of glaucoma. London, J \& A Churchill 1891 pp. 153, 158.

${ }^{18}$ Swan KC and Lindgren TW: Unintentional lens injury in glaucoma surgery. Trans Am Ophthalmol Soc 1980, 78: 55-69.

${ }^{19}$ Watson PG and Grierson I: The place of trabeculectomy in the treatment of glaucoma. Ophthalmology 1981, 88: 175-96.

${ }^{20}$ Watson PG: When to operate on primary open angle glaucoma. Eye 1987, 1: 51-4. 